\title{
Multiple Sclerosis: New Hypotheses Are Needed
}

\section{Konstantin E. Balashov*}

Department of Neurology, Rutgers-Robert Wood Johnson Medical School, USA

*Corresponding author: Konstantin E. Balashov, MD, PhD Department of Neurology, Rutgers-Robert Wood Johnson Medical School, 125 Paterson Street, 6th Floor, New Brunswick, NJ 08901, USA, Tel: 732-235-7733; E-mail: konstantin.balashov@rutgers.edu

Received date: May 22, 2014, Accepted date: May 23, 2014, Published date: May 26, 2014

Copyright: (c) 2014 Balashov KE, et al. This is an open-access article distributed under the terms of the Creative Commons Attribution License, which permits unrestricted use, distribution, and reproduction in any medium, provided the original author and source are credited.

\section{Short Communication}

Multiple Sclerosis (MS) was recognized as a distinct disease involving both the brain and spinal cord and associated with disseminated demyelinating plaques ("la sclérose en plaques") and axonal damage more than 145 years ago by French neurologist JeanMartin Charcot [1]. Since that time, the world has witnessed many remarkable discoveries. Due to progress in physics and mathematics, MRI has become a powerful tool in neurology allowing us to follow the progression of demyelinating lesions in living patients. Based on progress made in the field of immunology, a number of immunomodulatory drugs have been discovered, tested in clinical trials and become standards of care for the treatment of patients with relapsing-remitting form of MS. However, medications used in MS are called Disease-Modifying Treatments (DMTs) since they do not stop the disease but only delay its activity and progression. DMTs have limited clinical efficacy and may have significant adverse effects. At present, we do not know what causes MS and, therefore, we cannot develop the MS cure.

It has been well documented that MS pathogenesis involves both inflammation and neurodegeneration. Inflammation is more common in relapsing-remitting form of MS compared to primary-progressive form of MS and, in the past, was considered a primary feature of demyelination in MS. Surprisingly, myelin destruction has recently been reported to occur before inflammation at least in some patients [2]. It is believed that MS occurs as a result of a complex combination of genetic background and environmental factors. Studies show MS prevalence approximately 1 per 100,000 in certain areas of China, Japan and India, and approximately 10 per 100,000 in Africa, whereas numbers in Europe, Canada, and the United States are much higher For example, MS prevalence is 248 per 100,000 in Saskatoon, a city in central Saskatchewan, Canada [3]. In contrast to African Americans, Asian Americans continue to have low MS prevalence [4]. Therefore, the popular theory about the south-north gradient of MS prevalence has many limitations.

Apart from having close relatives with MS and demyelinating lesions found accidently on brain [5] or spine [6] MRI, other factors predicting the increased future risk of MS in healthy subjects have a limited appeal. The human leukocyte antigen $\mathrm{DRB1} 1^{\star} 1501$ haplotype is associated with both relapsing-remitting and primary-progressive forms of MS [7]. However, the DRB1 ${ }^{\star} 1501$ allele is highly expressed in both Caucasian and Asian populations [8]. The prevalence of MS is significantly increased among Epstein-Barr virus (EBV) seropositive subjects [9]. However, EBV seropositivity in Asia is not decreased compared to Western countries [10].

Current hypotheses addressing the cause of MS try to combine the generated data into likely explanations, but none has proved definitive. The most well-known hypothesis suggests that MS is an autoimmune process which leads to neuroinflammation and demyelination. The autoimmune hypothesis has existed for many years and is based on the model of experimental autoimmune encephalomyelitis (EAE) which is one of several animal models of demyelinating disease in the central nervous system (CNS). The main mechanism of EAE is an autoimmune T-cell-specific reaction to one of several myelin antigens present in the CNS. Several DMTs for MS (e.g., Glatiramer Acetate and Natalizumab), were developed from studies in EAE. However, the EAE model has a number of pitfalls in failing to predict the outcome of certain approaches in MS [11]. After many years of intensive studies and clinical trials, no myelin protein inducing autoimmune reaction in only MS patients has been confirmed. In addition, the autoimmune theory cannot explain neurodegenerative processes documented in the CNS of MS patients at the very early stage of the disease.

The second hypothesis suggests that MS is an infectious disease. For example, it was hypothesized that a certain virus might reactivate after years of latency and lyses oligodendrocytes in the CNS of patients with MS, as in progressive multifocal leukoencephalopathy, or initiate immunopathological demyelination, as in animals infected with Theiler's murine encephalomyelitis virus or coronaviruses [12]. However, no MS-specific virus has yet been isolated from the brains of patients.

The third hypothesis suggests that patients with MS have chronic cerebrospinal venous insufficiency (CCSVI) causing delayed venous outflow from the brain and spinal cord [13]. However, the initial promising results of Dr. Zamboni and coauthors could not be reproduced by other researchers. It was later reported that CCSVI occurs rarely in both patients with MS and in healthy people [14].

All three hypotheses mentioned above provide a reasonable explanation why the disease selectively involves the brain and spinal cord and does not affects other organs. However, they do not explain the key epidemiological finding of decreased MS prevalence among Asians. It is very likely that new hypotheses addressing the cause of MS are going to emerge in the near future. Therefore, two mandatory criteria are proposed for a new biological factor/mechanism hypothesized to be a MS cause:

Criterion 1. The new hypothesis has to explain why the disease is restricted to the CNS and causes both inflammation and neurodegeneration.

Criterion 2. The new hypothesis has to explain why the disease has low prevalence in Asia.

\section{References}

1. Charcot M (1868) Histologie de la sclérose en plaques. Gazette Hôpitaux.

2. Barnett MH, Prineas JW (2004) Relapsing and remitting multiple sclerosis: pathology of the newly forming lesion. Ann Neurol 55: 458-468. 
3. Rosati G (2001) The prevalence of multiple sclerosis in the world: an update. Neurol Sci 22: 117-139.

4. Langer-Gould A, Brara SM, Beaber BE, Zhang JL (2013) Incidence of multiple sclerosis in multiple racial and ethnic groups. Neurology 80: 1734-1739.

5. Lebrun C, Bensa C, Debouverie M, De Seze J, Wiertlievski S, et al. (2008) Unexpected multiple sclerosis: follow-up of 30 patients with magnetic resonance imaging and clinical conversion profile. J Neurol Neurosurg Psychiatry 79: 195-198.

6. Okuda DT, Mowry EM, Cree BA, Crabtree EC, Goodin DS, et al. (2011) Asymptomatic spinal cord lesions predict disease progression in radiologically isolated syndrome. Neurology 76: 686-692.

7. Barcellos LF, Sawcer S, Ramsay PP, Baranzini SE, Thomson G, et al. (2006) Heterogeneity at the HLA-DRB1 locus and risk for multiple sclerosis. Hum Mol Genet 15: 2813-2824.

8. Alcina A, Abad-Grau Mdel M, Fedetz M, Izquierdo G, Lucas $M$, et al. (2012) Multiple sclerosis risk variant HLA-DRB1*1501 associates with high expression of DRB1 gene in different human populations. PLoS One 7: e29819.
9. Ascherio A, Munch M (2000) Epstein-Barr virus and multiple sclerosis. Epidemiology 11: 220-224.

10. Takeuchi K, Tanaka-Taya K, Kazuyama Y, Ito YM, Hashimoto S, et al. (2006) Prevalence of Epstein-Barr virus in Japan: trends and future prediction. Pathol Int 56: 112-116.

11. Steinman L, Zamvil SS (2005) Virtues and pitfalls of EAE for the development of therapies for multiple sclerosis. Trends Immunol 26: 565-571.

12. Owens GP, Gilden D, Burgoon MP, Yu X, Bennett JL (2011) Viruses and multiple sclerosis. Neuroscientist 17: 659-676.

13. Zamboni P, Galeotti R, Menegatti E, Malagoni AM, Tacconi G, et al. (2009) Chronic cerebrospinal venous insufficiency in patients with multiple sclerosis. J Neurol Neurosurg Psychiatry 80: 392-399.

14. Traboulsee AL, Knox KB, Machan L, Zhao Y, Yee I, Rauscher A, et al. (2013) Prevalence of extracranial venous narrowing on catheter venography in people with multiple sclerosis, their siblings, and unrelated healthy controls: a blinded, case-control study. Lancet. 